\title{
INTENCIÓN EMPRENDEDORA DE ESTUDIANTES DE EDUCACIÓN MEDIA: EXTENDIENDO LA TEORÍA DE COMPORTAMIENTO PLANIFICADO MEDIANTE EL EFECTO EXPOSICIÓN"
}

\author{
Fabián Fernando Osorio** \\ Juan Carlos Londoño Roldán $n^{* * * * *}$
}

doi:10.11144/Javeriana.cao28-51.ieee. El artículo se recibió el 20/11/2013 y se aprobó el 30/05/2015. Sugerencia de citación: Osorio T., F. F. y J. C. Londoño R. (2015). Intención emprendedora de estudiantes de educación media: extendiendo la teoría de comportamiento planificado mediante el efecto exposición. Cuadernos de Administración, 28 (51), 103-131. http://dx.doi.org/10.11144/Javeriana.cao28-51.ieee

** Maestría en Administración de la Universidad del Valle, Cali, Colombia, 2010. Profesor de tiempo completo del Departamento Gestión de Organizaciones de la Pontificia Universidad Javeriana, Cali, Colombia. Correo electrónico: fosorio@javerianacali.edu.co

*** PhD de la University of Stirling, Scotland, U.K., 2013. Profesor de tiempo completo del Departamento Gestión de Organizaciones de la Universidad Javeriana, Cali, Colombia.

Correo electrónico:juanclondono@javerianacali.edu.co 


\section{Intención emprendedora de estudiantes de educación media: extendiendo la teoría de comportamiento planificado mediante el efecto exposición}

\section{RESUMEN}

El modelo de intención basado en la teoría del comportamiento planificado (TCP) ha sido evaluado empíricamente en la formación de intenciones emprendedoras, más que todo, en estudiantes universitarios de diferentes orígenes culturales. Este artículo prueba la TCP en una muestra de estudiantes de colegios de la ciudad de Cali, Colombia, a través de un modelo con cuadrados mínimos parciales y se encontró que la exposición previa en actividades de emprendimiento tiene un efecto positivo sobre los determinantes de la intención emprendedora. Esta línea de investigación se puede continuar con un estudio longitudinal que brindará resultados más sólidos y con mayor extensión de cara a las diferencias culturales.

Palabras clave: Intención emprendedora, teoría de comportamiento planificado, educación, efecto de exposición.

Clasificación JEL: L26, M13

\section{Entrepreneurial intention in middle and high school students: Using the exposure effect to extend the theory of planned behaviour}

\section{Intenção empreendedora de estudantes de educação média: estendendo a teoria de comportamento planejado mediante $o$ efeito exposição}

\section{ABstract}

The Theory of Planned Behavior (TPB) has been empirically tested in the creation of entrepreneurial intentions, above all, among university students of different cultural backgrounds. This article tests the TPB using a sample of middle and high school students in the city of Cali, Colombia, through a model with partial least squares and finds that the exposure to entrepreneurial activities has a positive effect on the determinants of entrepreneurial intention. This line of research can be continued with a longitudinal study that can bring extensive and more solid results in regards to cultural differences.

Keywords: Entrepreneurial intention, Theory of Planned Behavior, education, exposure effect.

JEL Classification: L26, M13

\section{Resumo}

O modelo de intenção baseado na teoria de comportamento planejado (TCP) tem sido avaliado empiricamente na formação de intenções empreendedoras, especialmente, em estudantes universitários de diferentes origens culturais. Este artigo prova a TCP numa amostra de estudantes de colégio da cidade de Cali, Colômbia, por meio de um modelo de mínimos quadrados parciais e constatou-se que a exposição prévia em atividades de empreendimento tem um efeito positivo sobre os determinantes de intenção empreendedora. Essa linha de pesquisa pode continuar com um estudo longitudinal que oferecerá resultados mais sólidos e com mais ênfase nas diferenças culturais.

Palavras-chave: intenção empreendedora, teoria do comportamento planejado, educação, efeito de exposição.

Classificação JEL: L26, M13 


\section{Introducción}

La economía del conocimiento, caracterizada por la ambigüedad y la incertidumbre, la globalización, la generación de nuevos saberes y la abundancia de información, requiere de individuos emprendedores (Julien, 2005). Los responsables del desarrollo de las políticas públicas sobre emprendimiento en Europa y Estados Unidos creen que es necesario un mayor nivel de cultura emprendedora para lograr altos niveles de crecimiento económico. Además, la investigación empírica soporta relaciones positivas entre la actividad emprendedora y los resultados económicos tales como el crecimiento económico y la innovación (2007). Los responsables del desarrollo de políticas públicas también creen que el aumento en los niveles de actividad emprendedora puede ser alcanzado a través de la educación (Commission, 2006). Los responsables de la política de educación en Colombia han propuesto una orientación general para construir una cultura del emprendimiento en los establecimientos de educación básica y media. En el marco normativo, la Ley 1014 de 2006, artículo 13, establece que el fomento de la cultura del emprendimiento y su formación en el nivel de educación básica y media es de carácter obligatorio. Pese a esta consideración poco se sabe sobre la forma en que se puede incentivar una cultura emprendedora en los establecimientos de educación básica y media.

Asimismo, nos enfrentamos a una de las preguntas predominantes en la investigación sobre emprendimiento que hace referencia al por qué algunas personas deciden ser emprendedoras mientras que otras no (Mitchell et al., 2007). Esta cuestión puede ser abordada en términos de los factores que influencian la decisión de emprender enfocandose en los rasgos o características de personalidad de los individuos (Shapero y Sokol, 1982). Recientemente en el campo de investigación de emprendimiento, los modelos de intención que se centran en las actitudes se han propuesto como una mejor explicación de dicha cuestión. En particular, es escaza la investigación de intención emprendedora en instituciones de educación en niveles de formación básica primaria y media secundaria, dejando un vacío en la literatura relacionada con el emprendimiento pre-universitario o de educación media (Peterman y Kennedy, 2003). Sin embargo, en años recientes han surgido estudios sobre el desarrollo de una cultura emprendedora en los establecimientos de educación de nivel básica y media (Donckels, 1991; Gasse, 1985; Kourilsky, 1995; Peterman y Kennedy, 2003) dado que se cree que el escenario ideal para adquirir los conocimientos básicos sobre una cultura emprendedora y fomentar una actitud positiva hacia la acción de emprender ocurre durante los años de la infancia y la adolescencia (Filion, 1994; Gasse, 1985). 
Comprender la intención emprendedora es de vital importancia en la predicción de la conducta. Las intenciones son un predictor importante del subsecuente comportamiento planificado (Baggozi et al., 1989) dado que la decisión de hacerse emprendedor puede considerarse voluntaria y consciente. En este contexto, la teoría de comportamiento planificado establece la premisa de que cualquier comportamiento requiere un cierto nivel de planeación y este puede ser predecido por la intención de adoptar dicho comportamiento (Ajzen, 1991). Así, el proceso de tomar la decisión de desarrollar una carrera emprendedora puede ser considerado como una conducta motivada o comportamiento planificado, existiendo una fuerte relación entre las intenciones y la acción. Con una comprensión de la intención emprendedora de los estudiantes de establecimeintos educativos de nivel media de educación, podemos predecir mejor si van a tomar medidas reales para acometer una acción emprendedora y de esta manera poder promover intenciones emprendedoras en los estudiantes de educación media, incrementando las posibilidades de que los estudiantes se involucren en emprendimiento.

En resumen, existe la necesidad de comprender de mejor manera la intención emprendedora en estudiantes de establecimientos educativos de nivel de educación media y cómo se afecta dicha intención. Dicha necesidad es el objetivo de este trabajo que se desarrolla en este orden: primero, se pone a prueba la suficiencia de la teoría de comportamiento planificado de Ajzen (1991) para predecir la intención emprendedora de los estudiantes de establecimientos educativos de nivel media en una muestra de estudiantes de colegios de la ciudad de Cali, Colombia. Y segundo, se evalúa cómo el efecto exposición afecta los determinantes de la intención emprendedora en estudiantes de nivel de educación media.

Este estudio tiene varias contribuciones y se espera que los resultados arrojen luces sobre una serie de cuestiones. Primero, poner a prueba la aplicabilidad del modelo de intención de Ajzen. Segundo, que sirva para dar claridad sobre la relación entre el efecto de exposición y los determinantes de la intención emprendedora. Y tercero, que el estudio ayude a avanzar en el conocimiento científico en el área de investigación de intencion emprendora mediante el reporte de resultados en una región/país que no ha sido incluida en investigaciones anteriores. Este hecho es de particular importancia teniendo en cuenta la insistencia de los investigadores en la necesidad de evaluar los modelos de intención en territorios con diferentes normas culturales y sociales, ya que este enfoque puede mejorar la comprensión actual de la validez y solidez del modelo de cara a marcadas diferencias culturales (Liñan y Chen, 2006; Brannback et al., 2007). 
Finalmente, los responsables de las politicas publicas y, en particular, los establecimientos de educación de nivel medio podrían obtener información útil en los resultados de esta investigación.

El artículo está organizado en cinco partes: (i) una revisión de la literatura sobre estudios previos que indagan la relación entre la intención emprendedora y la Teoría de Comportamiento Planificado; (ii) el planteamiento de las hipótesis; (iii) la metodología de la investigación; (iv) el análisis y los resultados, y (v) las conclusiones, implicaciones y limitaciones del estudio.

\section{Intención emprendedora y Teoría de Comportamiento Planificado}

La intención es un proceso necesario antes de llevar a cabo una acción. La investigación sobre comportamiento afirma que la intención tiene una capacidad de mayor explicación que otros factores, como por ejemplo, el carácter psicológico (Bird, 1988).

La intención emprendedora es un estado mental que las personas poseen en favor de optar por la creación de una nueva empresa o la creación de valor al interior de organizaciones existentes. Es el compromiso para desempeñar el comportamiento necesario para llevar a cabo una iniciativa emprendedora (Krueger, 1993). La investigación en este campo afirma que las intenciones son un predictor fundamental del comportamiento planeado (Baggozi et al., 1989; Kolvereid, 1996; Liñan, 2004). Por consiguiente, la intención emprendedora es un fenómeno importante de estudio. En primer lugary desde la mirada individual, con el fin de convertirse en fundadores de empresa, las personas deben convertirse primero en individuos emprendedores. Por lo tanto, el proceso que subyace a la aparición de la intención emprendedora y el comportamiento, es de suma importancia. Y en segundo lugar, desde lo económico, el informe de investigación Global Entrepreneurship Monitor (GEM) establece una correlación existente entre la tasa de crecimiento económico y el nivel y tipo de actividad emprendedora. En este sentido, el nivel de intención emprendedora refleja el potencial entorno económico de una región.

La intención emprendedora ha sido frecuentemente estudiada mediante el uso de modelos de intención; estos ofrecen un marco de trabajo coherente y robusto para la comprensión del proceso emprendedor. Los más utilizados en la literatura de emprendimiento son la teoría de comportamiento planificado (TCP) de Ajzen (1991) y el modelo social del evento emprendedor (SEE) de Shapero y Sokol (1982). La razón fundamental que justifica su uso reiterado es la capacidad que tienen los dos modelos de superponerse y su compatibilidad 
(Krueger et al., 2000). En consecuencia y de acuerdo con la investigación empírica, cada elemento de la TCP afecta de manera significativa a la intención emprendedora. En este sentido los resultados de la investigación soportan la aplicabilidad de la TCP en emprendimiento. En la Tabla 1 se presentan los estudios que han evaluado la eficacia del modelo TCP en el área de emprendimiento.

La forma en que están redactados los ítems de intención, suponen que el estudiante ya ha desarrollado en su mente la idea de crear empresa. Si responde positivamente a la afirmación significa que sí ha desarrollado esta idea, de lo contrario no la habría desarrollado. Como el modelo no es de tipo experimental no se induce la intención a los participantes sino que se apela a evaluar qué tanto se aproximan los mismos al comportamiento planteado.

\section{Tabla 1}

Comparación de estudios de Intención Emprendedora con TCP

\begin{tabular}{|c|c|c|c|}
\hline Autor (año) & Modelo de Intención & Muestra & Hallazgos \\
\hline $\begin{array}{l}\text { Kolvereid } \\
(1996)\end{array}$ & TCP & $\begin{array}{l}\text { Estudiantes noruegos } \\
\text { de escuela de negocios }\end{array}$ & $\begin{array}{l}\text { La experiencia de autoempleo, género y an- } \\
\text { tecedentes familiares solo afectan indirec- } \\
\text { tamente la intención de autempleo a través } \\
\text { de su efecto sobre la actitud, las normas } \\
\text { subjetivas y el control conductual percibido. }\end{array}$ \\
\hline $\begin{array}{l}\text { Tkachev y Kol- } \\
\text { vereid (1999) }\end{array}$ & TCP & $\begin{array}{l}\text { Estudiantes universita- } \\
\text { rios en Rusia }\end{array}$ & $\begin{array}{l}\text { La actitud, las normas subjetivas y el control } \\
\text { conductual percibido determina la intención } \\
\text { en la situación de elección de empleo de es- } \\
\text { tudiantes rusos. }\end{array}$ \\
\hline $\begin{array}{l}\text { Krueger et al. } \\
(2000)\end{array}$ & TCP / SEE & $\begin{array}{l}\text { Estudiantes universi- } \\
\text { tarios }\end{array}$ & $\begin{array}{l}\text { El modelo de intención es significativo, no en } \\
\text { tanto el determinante de las normas sociales. } \\
\text { Tanto TCPy SEE explican la intención empren- } \\
\text { dedora pero es mayor el de SEE. }\end{array}$ \\
\hline $\begin{array}{l}\text { Veciana et al. } \\
\text { (2005) }\end{array}$ & TCP/SEE & $\begin{array}{l}\text { Estudiantes universi- } \\
\text { tarios de Puerto Rico y } \\
\text { Cataluña }\end{array}$ & $\begin{array}{l}\text { La relación entre variables demográficas y la } \\
\text { intención emprendedora no es la misma con } \\
\text { estudiantes de diferentes países. }\end{array}$ \\
\hline $\begin{array}{l}\text { Liñan y Chen } \\
\text { (2006) }\end{array}$ & TCP & $\begin{array}{l}\text { Estudiantes universita- } \\
\text { rios de España y Taiwan }\end{array}$ & $\begin{array}{l}\text { Las variables demográficas, excepto el gé- } \\
\text { nero, tienen efecto con baja significatividad } \\
\text { sobre los determinantes de la intención em- } \\
\text { prendedora. }\end{array}$ \\
\hline $\begin{array}{l}\text { Fayolle et al. } \\
(2006)\end{array}$ & TCP & $\begin{array}{l}\text { Estudiantes universi- } \\
\text { tarios }\end{array}$ & $\begin{array}{l}\text { El programa de educación en emprendimiento } \\
\text { tiene impacto significativo sobre la intención } \\
\text { emprendedora de los estudiantes. Sin em- } \\
\text { bargo, el impacto no es significativo para el } \\
\text { comportamiento controlado percibido. }\end{array}$ \\
\hline $\begin{array}{l}\text { Kolvereid e } \\
\text { Isaksen (2006) }\end{array}$ & TCP & $\begin{array}{l}\text { Empresarios nacientes } \\
\text { de noruega }\end{array}$ & $\begin{array}{l}\text { Las actitudes y las normas subjetivas determi- } \\
\text { nan la intención de autoemplearse. }\end{array}$ \\
\hline
\end{tabular}




\begin{tabular}{|c|c|c|c|}
\hline Autor (año) & Modelo de Intención & Muestra & Hallazgos \\
\hline $\begin{array}{l}\text { Souitaris et al. } \\
(2007)\end{array}$ & TCP & $\begin{array}{l}\text { Estudiantes universita- } \\
\text { rios de Londres (UK) y } \\
\text { Grenoble (Francia) }\end{array}$ & $\begin{array}{l}\text { Los programas de emprendimiento son fuente } \\
\text { desencadenante que inspira a los estudiantes } \\
\text { a cambiar la mentalidad. }\end{array}$ \\
\hline Wu y Wu (2008) & TCP & $\begin{array}{l}\text { Estudiantes universita- } \\
\text { rios de China }\end{array}$ & $\begin{array}{l}\text { La educación universitaria ofrece una ex- } \\
\text { plicación para la intención emprendedora. } \\
\text { Se estima el modelo de TCP y se valida para } \\
\text { estudiantes universitarios de China. }\end{array}$ \\
\hline $\begin{array}{l}\text { Van Gelderen } \\
\text { et al. (2008) }\end{array}$ & TCP & $\begin{array}{l}\text { Estudiantes universita- } \\
\text { rios de Holanda }\end{array}$ & $\begin{array}{l}\text { Las variables más importantes para explicar } \\
\text { la intención emprendedora son el estar alerta } \\
\text { como expresión de la actitud y la importancia } \\
\text { de la seguridad financiera como expresión } \\
\text { del comportamiento controlado percibido. }\end{array}$ \\
\hline
\end{tabular}

Fuente: elaboración propia.

\section{Hipótesis}

\subsection{Modelo de intención emprendedora}

La teoría de comportamiento planificado (TCP) es un modelo general para explicar el comportamiento individual; este ha sido muy útil para la evaluación de la intención emprendedora en el marco de la investigación en emprendimiento (Krueger et al., 2000). La TCP plantea que las acciones del individuo están precedidas por decisiones conscientes para actuar de una manera determinada. Además establece que esas intenciones son el resultado de actitudes presentadas en virtud de las experiencias de vida, las características personales y las percepciones construidas por el individuo a partir de experiencias pasadas. Según Ajzen (1991) hay tres determinantes de la intención para un comportamiento:

1. Actitud hacia el comportamiento: se entiende como el grado con el cual un individuo tiene una valoración favorable o desfavorable del comportamiento en cuestión, en este caso de llevar a cabo una acción emprendedora. Es un reflejo de las creencias y opiniones que posee el individuo sobre dicho comportamiento.

2. Normas subjetivas: hacen referencia a la presión social percibida para realizar o no una conducta determinada. Hace referencia al grado con el cual el comportamiento a realizar cumple con los deseos de aquellos individuos importantes en la vida del individuo. Por ejemplo, las expectativas de la familia del individuo sobre la conveniencia de que se haga empresario. 
3. Percepción de control del comportamiento: hace referencia a la facilidad o dificultad percibida de la realización de un comportamiento determinado. Es la percepción que tiene el individuo de su habilidad para llevar a cabo una conducta específica. Este determinante está estrechamente relacionado con el concepto de autoeficacia (Ajzen, 1987) desarrollado por Bandura (1986). Ambos refieren a los factores perceptuales que son específicos para la consecución de un determinado comportamiento u objetivo conductual. Para este estudio la pregunta hecha a los estudiantes se relacionaba con la capacidad percibida por ellos para llevar a cabo cualquier tipo de acción emprendedora la cual se circunscribe a actividades que impliquen trabajo, recompensa, riesgo, nuevas formas de hacer y otros aspectos alineados con el concepto de proyecto/empresa (Bird, 1988).

Como se planteó, la TCP y el modelo social del evento emprendedor (SEE) presentan un alto nivel de compatibilidad mutua. El determinante de Actitud hacia el Comportamiento abarca la noción de deseabilidad percibida del modelo SEE; las Normas Subjetivas coinciden con la noción de deseabilidad y factibilidad y la factibilidad coincide con la Percepción de control de comportamiento (Krueger et al., 2000). En consecuencia, ambos modelos enfatizan los antecedentes motivacionales para la intención emprendedora.

Las variables utilizadas por la TCP reflejan en buena parte lo que otros modelos como el de competencias pretenden explicar. Si partimos desde un análisis psicológico de las competencias podemos observar como de acuerdo con McClelland (1973), las competencias se definen como motivaciones y rasgos de personalidad, en tanto McClelland y Boyatzis (1980) las definen como un cuerpo genérico de conocimientos, motivos, rasgos, autoimágenes y roles sociales que están relacionadas con un desempeño superior en el trabajo. Las motivaciones hacen parte de las actitudes, los roles sociales hacen parte de las normas subjetivas, mientras que la autoimagen y habilidades hacen parte de las percepciones de control.

Además, la TCP es un elemento valioso para la comprensión del proceso de creación de nuevas empresas independiente de las diferencias culturales. El asunto es si este modelo sirve como un modelo cognitivo apropiado para influenciar a los estudiantes de nivel de educación media hacia el emprendimiento, y en lo cual existe muy poca evidencia empírica. Por lo tanto, en referencia al modelo TCP de Ajzen, proponemos las siguientes hipótesis:

H1: La actitud personal hacia el emprendimiento está positivamente relacionada con la intención emprendedora de estudiantes de nivel de educación media. 
H2: La norma subjetiva está positivamente relacionada con la intención emprendedora de estudiantes de nivel de educación media.

H3: La percepción de control de comportamiento está positivamente relacionada con la intención emprendedora de estudiantes de nivel de educación media.

\subsection{El efecto de exposición previa y los determinantes} de la intención emprendedora

Krueger et al. (2000) argumentan que en virtud de que las variables exógenas y demográficas operan de manera indirecta sobre la intención emprendedora generalmente no se incluyen en los modelos de intención. Sin embargo, otros investigadores enfatizan la necesidad de incorporar este tipo de variables para comprender mejor la intención emprendedora. Como lo plantean Van Gelderen et al. (2008), variables adicionales como el género, la experiencia laboral, el rol de los modelos familiares y los rasgos de personalidad mejoran el entendimiento de la intención emprendedora. La importancia de la influencia de la exposición previa a actividades emprendedoras sobre la intención emprendedora es respaldada por algunos estudios (Drennan et al., 2005; Harris y Gibson, 2008), sin embargo es aún escaso el uso del modelo de TCP con referencia a la relación de exposición de actividades emprendedoras y la intención emprendedora en estudiantes de nivel de educación media. El modelo de TCP asume que las experiencias previas influenciarán las intenciones indirectamente a través de las actitudes, las normas sociales y el control percibido por el individuo (Ajzen, 1991), así, una alta frecuencia de exposición deberá ser un mejor predictor de actitudes hacia el inicio de una acción emprendedora (Krueger, 1993). Además, la teoría de efecto de exposición plantea que la mera exposición repetida del individuo a un objeto de estímulo mejora su actitud hacia este (Zajonc, 1968). Teniendo en cuenta que las actitudes están abiertas al cambio, las actitudes emprendedoras pueden ser influenciadas por la exposición previa a frecuentes actividades emprendedoras, por lo tanto, se plantea la siguiente hipótesis:

H4: La exposición previa de los estudiantes de nivel de educación media con actividades de emprendimiento afecta las actitudes personales que determinan la intención emprendedora.

En la TCP, las normas subjetivas miden la presión social percibida para llevar a cabo o no, una acción emprendedora. En general, los estudiantes están en una etapa de búsqueda de sus preferencias, en la decisión de una carrera y la opinión de padres, familiares y ami- 
gos es importante. Las expectativas de su familia pueden influenciar de manera positiva o negativa la conveniencia de que se convierta en empresario. Sin embargo, ¿cómo la exposición previa a actividades emprendedoras puede afectar este aspecto afectivo? En este sentido, nuevamente la teoría del efecto de exposición constituye un marco teórico pertinente para comprender la relación de la mera exposición y el afecto (Zajonc, 1968). La investigación del efecto de exposición se ha enfocado con mayor frecuencia sobre los efectos actitudinales (Harrison, 1977), sin embargo también existe evidencia que los efectos de exposición pueden ocurrir en los aspectos afectivos, es decir, que dichos efectos pueden ocurrir en las percepciones de las personas.

Las preferencias (que no son más que percepciones) constituyen una de las fuentes fundamentales del cambio y estabilidad social e individual. Estas le dan dirección a nuestras vidas y significado a nuestras acciones, pero sobre todo son capaces de influenciar valores ideológicos, compromisos políticos y normas culturales/sociales (Zajonc, 1968). En el marco de la teoría del efecto de exposición la forma en que las preferencias son adquiridas es justamente con la exposición frecuente a un estímulo (Zajonc, 2001). Frente a la exposición previa están los eventos que se caracterizan por una notable ausencia de consecuencias nocivas o adversas. Por lo tanto, la misma ausencia de una consecuencia adversa bien podría actuar como un estímulo no condicionado, constituyendose en una señal de seguridad que está asociada al estímulo condicionado. Después de una ocurrencia repetida al estímulo condicionado hay una asociación del estímulo no condicionado, que es simplemente el hecho de que el individuo no sufre ninguna consecuencia adversa, comunicando una respuesta de que el entorno actual es seguro. Así, la experiencia repetitiva en sí misma es capaz de producir un estado afectivo positivo y por tanto cambiar las percepciones del individuo (Zajonc, 2001). La investigación empírica muestra que la exposición repetida benigna puede en sí misma mejorar las percepciones, y que tal efecto puede ser unido no sólo a los estímulos que han sido expuestos, sino tambien a estímulos semejantes y/o distintos a los que ya haya experimentado.

Si los estudiantes de nivel de educación media se exponen de manera frecuente a la realización de actividades emprendedoras se esperaría que dicha exposición genere un efecto positivo sobre la percepción de las normas sociales. Por lo anterior, se propone una hipótesis adicional:

H5: La exposición previa de los estudiantes de nivel de educación media con actividades de emprendimiento afecta las normas subjetivas que determinan la intención emprendedora. 
En cuanto a la actividad emprendedora, la percepción de control de comportamiento se refiere a la percepción de la facilidad o dificultad que posee el individuo en llevar a cabo una acción emprendedora. En analogía con la autoeficacia, se refiere a los juicios o creencias de los individuos sobre su capacidad para organizar y seguir cursos de acción para obtener un resultado (Bandura, 1986). Dicha percepción de facilidad o dificultad, así como los juicios y creencias sobre la capacidad de llevar a cabo una acción son adquiridos gradualmente mediante el desarrollo de complejas habilidades cognitivas, sociales, linguisticas y/o físicas, que son logradas a través de la experiencia (Bandura, 1982; Gist, 1987). Así, la adquisición de habilidades a través de logros del pasado mediante la exposición previa, refuerza la percepción de control de comportamiento y contribuye a mayores aspiraciones y rendimiento futuro (Herron y Sapienza, 1992). La formación de autoeficacia/percepción de control de comportamiento, tambien se ve influenciada por la evaluación individual de la disponibilidad de recursos y limitaciones personales, que pueden afectar el rendimiento futuro (Ajzen, 1987). La manera más eficaz para que las personas desarrollen un fuerte sentido de percepción de control del comportamiento es através de experiencias repetidas de dominio o logros de desempeño (Bandura, 1982; Gist, 1987; Wood y Bandura, 1989). Por lo tanto, se espera que la exposición previa de los estudiantes en actividades de emprendimiento aumente la percepción de su capacidad de llevar a cabo una acción emprendedora y mantenga su esfuerzo aunque los resultados esperados sean inciertos. Resulta así la siguiente hipótesis:

H6: La exposición previa de los estudiantes de nivel de educación media con actividades de emprendimiento afecta la percepción de control del comportamiento que determina la intención emprendedora.

Hasta aquí se ha abordado el efecto de exposición sobre los determinantes de la intención emprendedora. La intención es un elemento central en la TCP y en ella se capturan los factores motivacionales que influyen en la conducta e indican cuándo el individuo está dispuesto a probar un comportamiento o la cantidad de esfuerzo que está dispuesto a ejercer para realizar el comportamiento. Por lo tanto, en la ejecución de acciones emprendedoras por parte de los estudiantes, son importantes las intenciones como variable de la conducta.

En suma, las hipótesis $\mathrm{H} 1, \mathrm{H} 2$ y H3 describen e intentan validar el modelo de intención de acuerdo a la TCP. En tanto las hipótesis $\mathrm{H} 4, \mathrm{H} 5$ y H6 describen el efecto sobre los determinantes propuestos por la TCP de la intención emprendedora. Todas las hipótesis establecen el modelo teórico propuesto en este estudio y que se presenta en la Figura 1. 


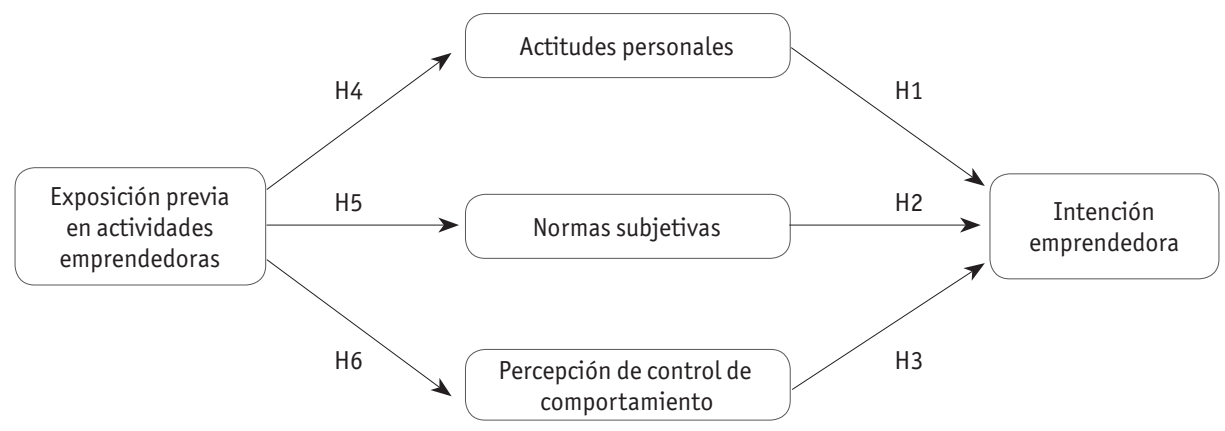

Figura 1. Modelo teórico propuesto

Fuente: elaboración propia.

\section{Metodología}

Los datos de este estudio se obtuvieron de una muestra de 643 estudiantes (260 hombres y 383 mujeres) de los grados décimo $(n=268)$ y once $(n=375)$ de establecimientos educativos de nivel de educación media y básica públicas y privadas de la ciudad de Cali y sus alrededores. Los estudiantes se encuentran entre los 15 y 17 años de edad ( $n=512$; $82,7 \%)$, se encuentran ubicados en los estratos socioeconómicos 1 ( $n=122 ; 19 \%), 2$ $(n=200 ; 31,1 \%)$ y $3(n=190 ; 29,5 \%)$ y asisten en su mayoría a la jornada de la mañana $(n=504 ; 78,4 \%)$. Los datos fueron recolectados mediante una encuesta estructurada la cual fue aplicada por un equipo de personas capacitadas para tal fin, a los estudiantes en sus lugares de estudio de manera personal.

La medición de las variables de la TCP se realizó teniendo en cuenta los principios generales de Ajzen (1991) y la variable exposición se midió considerando la teoría de efecto de exposición de Zajonc (1968). Las preguntas utilizadas se presentan en el Anexo 1. El comportamiento objetivo de la investigación es crear su propia empresa o, en otras palabras, ser empresario.

Las normas subjetivas se construyeron multiplicando las creencias normativas por la motivación para cumplir. El producto de estas dos se utilizó como insumo para evaluar la importancia de los influenciadores en la norma social. En este caso, la medición indirecta de las normas subjetivas sustituye a la medición directa de las mismas. Dado que no todas las personas (padres, profesores, amigos) influyen de la misma forma en las normas subjetivas, se dibuja las normas subjetivas (NS) como un constructo formativo. 
Las actitudes miden si para el joven estudiante ser emprendedor es algo positivo, una actividad que les resulta más atractiva que otras alternativas de vida.

Las normas subjetivas miden la opinión que tienen los diferentes entes normativos (padres, hermanos, abuelos, parientes, profesores, etc.) con respecto a ser empresario y la importancia que tiene para el joven cada una de estas personas, que vienen a ser referentes sociales.

La percepción de control de comportamiento ( $\mathrm{PBC}$ ) mide la percepción que tiene los jóvenes sobre su propia capacidad para ejecutar acciones emprendedoras, como proponer soluciones novedosas, realizar proyectos y tomar decisiones.

La intención emprendedora está medida como la claridad que tiene el joven sobre su propósito de ser emprendedor. No es suficiente querer ser emprendedor, sino que debe tener una visión clara de lo que quiere lograr.

La exposición mide la frecuencia con la que el joven realiza actividades que lo vinculan con la actividad emprendedora. Es decir, aprende a ser emprendedor haciendo actividades relacionadas con el emprendimiento, como identificar oportunidades empresariales o proyectos empresariales.

Es pertinente utilizar la técnica de modelación de ecuaciones estructurales (SEM) que más se ajuste a los objetivos de investigación, las características de los datos y las características del modelo (Fornell y Bookstein, 1982; Gefen et al., 2011; Hair et al., 2011; Reinartz et al., 2009). En este caso el objetivo del estudio es confirmar una teoría. Para este caso CB-SEM podría ser una opción, sin embargo, no es el más adecuado en cuanto a las características del modelo, pues se debe considerar si este es reflectivo o formativo; esto es un elemento importante a considerar en los modelos SEM (Diamantopoulos y Winklhofer, 2001; Jarvis et al. , 2003). El uso de CB-SEM para modelos formativos aplica solo bajo ciertas condiciones (Bollen y Davis, 2009; Diamantopoulos et al. , 2008) mientras el uso de PLS-SEM aplica en forma irrestricta tanto para modelos reflectivos como formativos (Chin, 1998).

Desde el punto de vista teórico, para decidir si un modelo es formativo o reflectivo es importante considerar la naturaleza del constructo, su causalidad y las características de sus indicadores. En este estudio, las normas subjetivas tienen una particularidad y es que los diferentes tipos de personas evaluadas (padres, familiares, etc.) pueden tener diferentes opiniones sobre el emprendimiento, por lo tanto su naturaleza no es reflectiva. 
Utilizar las actitudes como una medida reflectiva es generalmente aceptado, pero en el caso de las normas subjetivas aún existe controversia (Armitage y Conner, 2001; Hagger et al., 2002) especialmente dados los resultados contradictorios que ha obtenido esta medida (Armitage y Conner, 2001). En el campo del emprendimiento (Li et al., 2008) y otros estudios como el Global Entrepreneurship Monitor (GEM), han incluido las normas sociales, además, algunos estudios recientes sugieren que las normas sociales pueden ser medidas en modo formativo (Rhodes y Courneya, 2003).

\section{Resultados}

Las hipótesis fueron evaluadas usando un modelo de ecuaciones estructurales (SEM), específicamente, se realizó una aproximación con cuadrados mínimos parciales (PLS). El software usado fue Smart PLS (Ringle et al., 2005). Este software permitió la construcción del modelo en donde se evaluaron las relaciones entre los constructos.

La evaluación del modelo en PLS sigue dos pasos: el primero consiste en la medición del modelo externo o modelo de medición. El propósito de esta etapa es determinar la confiabilidad y validez del modelo. Las cargas de los indicadores deben ser superiores a 0,7 . En estudios exploratorios cargas superiores a 0,4 son aceptables (Hulland, 1999). Como se puede observar en la Tabla 2, todos los indicadores de este estudio alcanzaron y sobrepasaron en su mayoría el nivel de 0,7 requerido.

\section{Tabla 2}

Cargas, pesos, confiablidad compuesta y varianza extraída media

\begin{tabular}{cllccc}
\hline Constructo & \multicolumn{1}{c}{ Item } & Carga & Peso & CR & AVE \\
\hline \multirow{4}{*}{ AT } & Acudisnece & 0,83 & & 0,89 & 0,67 \\
& Acuporecur & 0,82 & & & \\
& Acuprefeemp & 0,71 & & & \\
& Acutodoposi & 0,89 & & & N/A \\
& Padres & & 0,79 & N/A & \\
& Abuelos & & 0,17 & & \\
& Parientes & & 0,20 & & \\
& Hermanos & & $-0,23$ & & \\
& Amigos & & 0,37 & & \\
& Profesores & & 0,12 & & \\
& Conocidos & & $-0,06$ & & \\
& & & & & \\
\end{tabular}




\begin{tabular}{cllccc}
\hline Constructo & \multicolumn{1}{c}{ Item } & Carga & Peso & CR & AVE \\
\hline \multirow{3}{*}{ PBC } & Capacipropo & 0,75 & & 0,79 & 0,56 \\
& Capamotivar & 0,79 & & & \\
& Capatomad & 0,70 & & & \\
\hline \multirow{3}{*}{ EXP0 } & freempleo & 0,82 & & 0,87 & 0,63 \\
& freinvestigo & 0,84 & & \\
& freresuelvo & 0,73 & & & \\
& fretrabajo & 0,79 & & 0,93 \\
\multirow{2}{*}{ INTENT } & acuclarocrea & 0,92 & & \\
& acuclaronove & 0,92 & & & \\
& acuclaropas & 0,87 & & & \\
\hline
\end{tabular}

Fuente: elaboración propia.

En la Tabla 2 también se presentan indicadores de la calidad de los datos como la confiabilidad compuesta (CR) y la varianza extraída media (AVE). La CR es una medida de la consistencia interna de los datos y debe ser igual o mayor a 0,7 (Nunnally, 1978). La AVE es una medida de la validez discriminante de los datos y mide el grado en que dos medidas del mismo concepto están correlacionadas. El valor mínimo de AVE debe estar por encima de 0,5 (Fornell y Larcker, 1981).

En este estudio todos los valores de la AVE se encontraron por encima del 0,5 y lo mismo sucedió con los valores de CR, que se encontraron por encima de 0,7; garantizando así la calidad de los datos.

Se comparan también la raíz cuadrada de la AVE con las correlaciones entre los constructos reflectivos. En la Tabla 3 se puede observar que el valor de la raíz cuadrada de la AVE es superior a los elementos que se encuentran por fuera de la diagonal en la misma fila y columna (Chin, 1998). Todos los constructos evaluados se correlacionaron con su mismo constructo más que con cualquier otro, alcanzando así una buena validez discriminante.

La validez discriminante también fue evaluada a nivel de ítem. El procedimiento para determinar este tipo de validez consiste en comparar las cargas de cada ítem contra su propio constructo en comparación con la carga que tiene contra otras variables. En la Tabla 4 se presentan los resultados de validez discriminante alcanzados en este estudio. Todos los valores presentaron una carga que es más grande con sus propios ítems que con otros indicadores. 


\section{Tabla 3}

Correlación de las variables latentes versus raíz cuadrada de la AVE

\begin{tabular}{ccccc}
\hline & ATT & EXPO & INTENT & PBC \\
\hline ATT & 0,82 & & & \\
EXPO & 0,40 & 0,80 & & \\
INTENT & 0,61 & 0,58 & 0,90 & \\
PBC & 0,23 & 0,44 & 0,37 & 0,76 \\
\hline
\end{tabular}

Fuente: elaboración propia.

\section{Tabla 4}

Cargas cruzadas

\begin{tabular}{lcccc}
\hline \multicolumn{1}{c}{ ITEM LEVEL } & ATT & EXPO & INTENT & PBC \\
\hline INTENT acuclarocrea & 0,58 & 0,52 & 0,92 & 0,33 \\
INTENT acuclaronove & 0,55 & 0,53 & 0,92 & 0,33 \\
INTENT acuclaropas & 0,53 & 0,52 & 0,87 & 0,35 \\
ATT acudisnece & 0,83 & 0,34 & 0,49 & 0,26 \\
ATT acuoporecur & 0,82 & 0,29 & 0,50 & 0,13 \\
ATT acuprefeemp & 0,71 & 0,26 & 0,36 & 0,15 \\
ATT acutodoposi & 0,89 & 0,39 & 0,62 & 0,19 \\
PBC capacipropo & 0,15 & 0,32 & 0,27 & 0,75 \\
PBC capamotivar & 0,22 & 0,36 & 0,31 & 0,79 \\
PBC capatomadeci & 0,13 & 0,32 & 0,23 & 0,70 \\
EXPO freempleo & 0,38 & 0,82 & 0,51 & 0,36 \\
EXPO freinvestigo & 0,37 & 0,84 & 0,51 & 0,39 \\
EXPO freresuelvo & 0,21 & 0,73 & 0,37 & 0,34 \\
EXPO fretrabajo & 0,28 & 0,79 & 0,42 & 0,33 \\
\hline
\end{tabular}

Fuente: elaboración propia.

Segundo paso: evaluación del modelo estructural, modelo interno

Una vez establecida la viabilidad de las medidas externas, pasamos a ofrecer evidencias sobre la confiabilidad del modelo interno. El algoritmo de PLS estimó la varianza explicada $\left(\mathrm{R}^{2}\right)$ para las cuatro variables dependientes. La fuerza del modelo teórico fue establecida por dos factores: la $R^{2}$ y el nivel de significancia de los caminos estructurales. Las varianzas explicadas $\left(R^{2}\right)$ son presentadas en la Tabla 5. 


\section{Tabla 5}

Varianza explicada de variables latentes

\begin{tabular}{cc}
\hline & R Cuadrado \\
\hline ATT & 0,16 \\
INTENT & 0,44 \\
PBC & 0,20 \\
SN & 0,08 \\
\hline
\end{tabular}

Fuente: elaboración propia.

Falk y Miller (1992) sugieren que el valor de la $\mathrm{R}^{2}$ debe ser superior a 0,1. Esta recomendación se cumple para todas las varianzas explicadas del modelo con la excepción de SN que solo llegó a un 0,08. El bajo nivel de varianza explicada alcanzado por la norma subjetiva indica que esta variable explica muy poco la intención emprendedora.

El nivel de significancia de las hipótesis fue evaluado usando el método de Bootstrap. Se seleccionaron 5000 sub-muestras, un valor superior al número de observaciones válidas en este estudio (643).

La Tabla 6 presenta los coeficientes y el nivel de significancia de los caminos evaluados. Todos los caminos son significativos y sus coeficientes fueron positivos. La única excepción es el camino que va de las normas subjetivas a la intención que presentó un coeficiente positivo pero bajo y fue significativo pero no con un $99 \%$ de confianza como los otros, sino con un $90 \%$ de confianza.

\section{Tabla 6}

Coeficientes, error estándar, estadística t y significancia

\begin{tabular}{rcccc}
\hline & Coeficiente & Error estandar & Estadísticot & Significancia \\
\hline ATT -> INTENT & 0,53 & 0,04 & 14,09 & $* * *$ \\
EXPO -> ATT & 0,39 & 0,03 & 11,26 & $* * *$ \\
EXPO -> PBC & 0,44 & 0,03 & 12,25 & $* * *$ \\
EXPO -> SN & 0,29 & 0,04 & 7,62 & $* * *$ \\
PBC -> INTENT & 0,23 & 0,03 & 7,02 & $* *$ \\
SN -> INTENT & 0,07 & 0,04 & 1,90 & $*$ \\
\hline
\end{tabular}

${ }^{*}=90 \%$ significancia valor- $\mathrm{t}=1,64 .{ }^{* *}=95 \%$ significancia valor- $\mathrm{t}=1,96 .{ }^{* * *}=99 \%$ significancia valor- $\mathrm{t}=2,58$. Fuente: elaboración propia. 


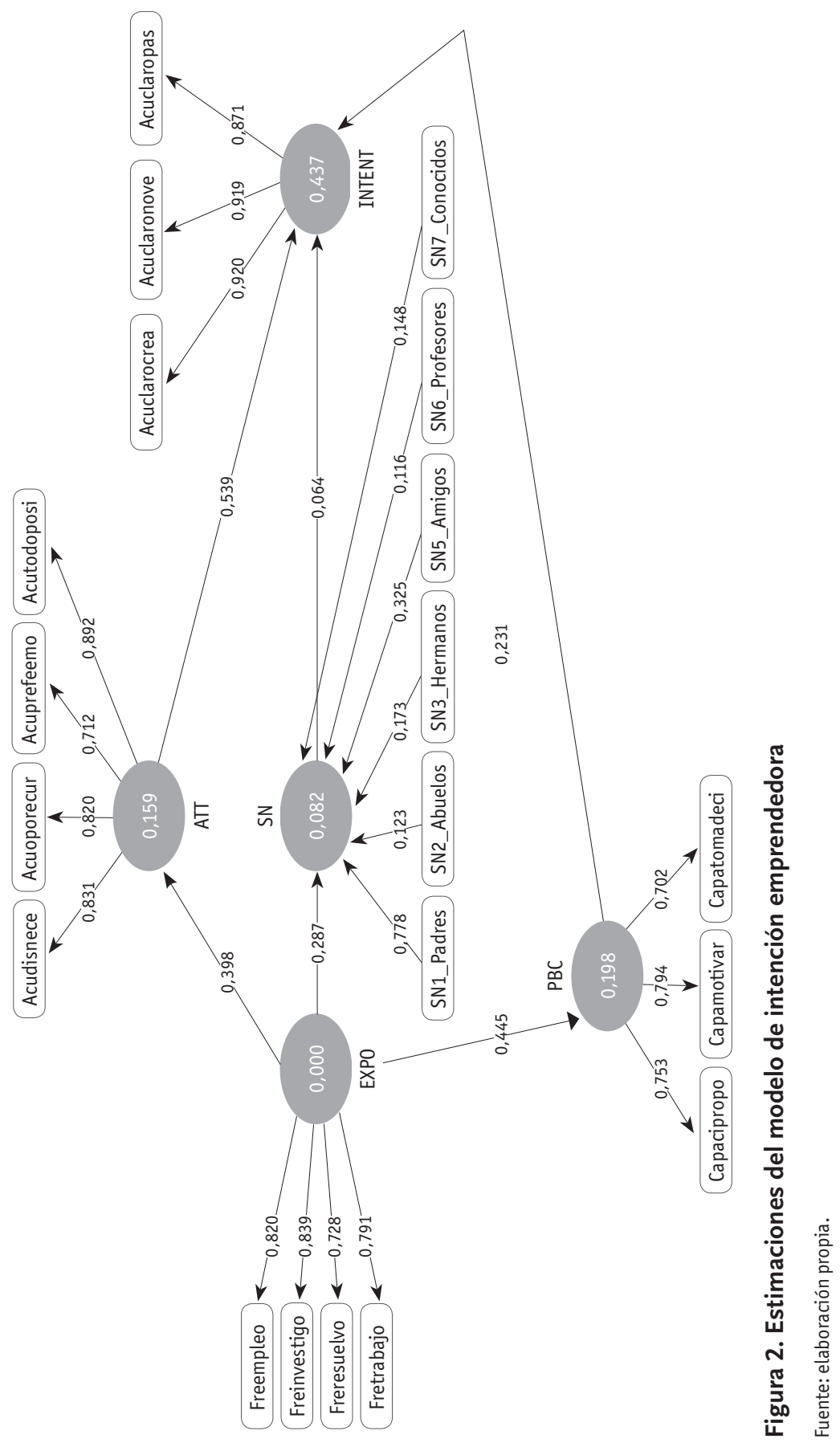


Relevancia de la predicción: Blindfolding. Se utilizó la técnica de reutilización de la muestra predictiva (blindfolding) desarrollada por Stone (1974) y Geisser (1975). Se seleccionó el número siete como número de distancia de omisión. Se calculó la relevancia predictiva relativa utilizando la ecuación 1.

Ecuación 1: Impacto relativo del modelo estructural

Ecuación $1-q^{2}$ : Impacto relativo del modelo estructural

$$
\left.\left.q^{2}=\left[Q^{2} \text { (incluido }\right)-Q^{2}(\text { excluido })\right] / 1-Q^{2} \text { (incluido }\right)
$$

Fuente: Chin (2010).

Los valores de la relevancia de la predicción relativa $\left(q^{2}\right)$ alcanzada por este estudio pueden ser encontrados en la Tabla 7.

Tabla 7

Relevancia de predicción $\left(q^{2}\right)$

\begin{tabular}{rcccc}
\hline & q Incl & q Exclu & 1-q Incl & q2 \\
\hline ATT -> INTENT & 0,35 & 0,15 & 0,65 & 0,31 \\
PBC -> INTENT & 0,35 & 0,31 & 0,65 & 0,06 \\
SN -> INTENT & 0,35 & 0,35 & 0,65 & 0,00 \\
\hline
\end{tabular}

Fuente: elaboración propia.

Los valores de 0,$02 ; 0,15$ y 0,35 (débil, mediano y largo respectivamente) fueron usados para interpretar los resultados. Según este resultado, las actitudes tuvieron un nivel de relevancia alto para la predicción, mientras la PBC fue baja y el de las normas subjetivas fue nulo.

Tamaño del efecto. El tamaño del efecto mide el nivel de impacto que tiene la variable independiente sobre la variable dependiente. El tamaño del efecto fue calculado usando la fórmula 2.

Fórmula 2: Tamaño del efecto

$$
f^{2}=\left[R^{2} \text { (incluido) }-R^{2} \text { (excluido) }\right] / 1-R^{2} \text { (incluido) }
$$

Fuente: Chin (2010). 
Para evaluar el tamaño del efecto, un $\mathrm{f}^{2}$ de 0,$02 ; 0,15$ y 0,35 representan un efecto pequeño, mediano y grande respectivamente (Cohen, 1988). Se utilizan estos valores porque en el contexto de la teoría de comportamiento planeado, Sutton (1998) tras revisar nueve meta-análisis de dicha teoría, encontró que generalmente se obtienen efectos medianos a grandes de acuerdo con la clasificación de Cohen. Sutton también encontró que estos modelos explican entre un $40 \%$ y un $50 \%$ de la varianza en la intención. En el mismo sentido Armitage y Conner (2001) encontraron que la TCP da cuenta de un 39\% de la varianza en la intención. De acuerdo con estos resultados, la $\mathrm{R}^{2}$ de este estudio $(0,44)$ se encuentra cercana a los valores encontrados en otros estudios de la TCP. Los resultados del tamaño del efecto para este estudio se presentan en la Tabla 8.

\section{Tabla 8}

Tamaño del efecto

\begin{tabular}{ccccc}
\hline PATH & R Incl & R Exclu & 1-R incl & f2 \\
\hline ATT -> INTENT & 0,43 & 0,18 & 0,57 & 0,44 \\
PBC -> INTENT & 0,43 & 0,38 & 0,57 & 0,09 \\
SN -> INTENT & 0,43 & 0,43 & 0,57 & 0,00 \\
\hline
\end{tabular}

Fuente: elaboración propia.

Al igual que con el nivel de predicción, el efecto de las actitudes sobre la intención fue alto, el de la PBC fue bajo y en de la SN fue nulo.

Análisis del constructo formativo (SN). No se espera que los indicadores de los constructos formativos correlacionen unos con otros. Por lo tanto, las medidas tradicionalmente usadas para medir su validez no son las más apropiadas (Chin, 1998). Para proporcionar evidencia de la validez nomológica o externa, se presentan los pesos y el grado de multicolinealidad. La multicolinealidad se puede evaluar por medio de factor de inflación de la varianza (VIF) el índice de condición y el nivel de significancia de los pesos. El nivel de VIF debe ser menor que 5 y la tolerancia mayor a 0,2; y el índice de condición menor a 30 (Hair et al., 2011).

En todos los casos el índice de condición fue inferior a 30. El nivel de significancia fue alcanzado por padres, abuelos y amigos, pero no fueron significativos hermanos, parientes, profesores y conocidos. El nivel de tolerancia fue superior a 0,2 en todos los indicadores e igualmente se obtuvieron niveles menores a 5 en el FIV. 


\section{Tabla 9}

Índice de condición, estadística $t$, significancia, tolerancia y VIF

\begin{tabular}{lccccc}
\hline & Índice de condición & $\mathrm{t}$ & Sig. & Tolerancia & VIF \\
\hline SN1 Padres & 4,64 & 5,96 & 0,00 & 0,87 & 1,15 \\
SN2 Abuelos & 5,64 & 2,24 & 0,03 & 0,74 & 1,36 \\
SN3 Hermanos & 5,90 & 1,57 & 0,12 & 0,88 & 1,14 \\
SN4 Parientes & 8,29 & $-1,44$ & 0,15 & 0,46 & 2,18 \\
SN5 Amigos & 9,28 & 2,30 & 0,02 & 0,48 & 2,08 \\
SN6 Profesores & 10,29 & 0,72 & 0,47 & 0,51 & 1,98 \\
SN7 Conocidos & 13,23 & $-0,64$ & 0,53 & 0,35 & 2,83 \\
\hline
\end{tabular}

Fuente: elaboración propia.

\section{Conclusiones, implicaciones y limitaciones}

En este estudio, el cual se basa en la teoría de comportamiento planificado, el modelo con cuadrados mínimos parciales muestra que la intención emprendedora de estudiantes de nivel de educación media de una muestra en instituciones de la ciudad de Cali, Colombia, puede ser explicado por las actitudes personales y la percepción del control de comportamiento. Para los estudiantes de nivel de educación media la intención está determinada por la percepción de la conveniencia de desarrollar una carrera empresarial, sin embargo, como otros estudios lo han planteado (Krueger et al., 2000; Veciana et al., 2005), dicha percepción de conveniencia no viene dada por las normas subjetivas. Este resultado obtenido, se encuentra en línea con la investigación en el área de TCP, que en diferentes meta-análisis reportan un efecto generalmente débil y/o nulo (Sheppard et al., 1988; Van den Putte, 1991; Sparks et al., 1995; Godin y Kok, 1996; Armitage y Conner, 2001). Estos establecen que el componente de normas subjetivas raramente predice las intenciones y algunos investigadores incluso lo remueven de los análisis. Frente a este resultado el mismo Ajzen (1987) explica que este resultado puede ocurrir cuando los individuos cuentan con un alto nivel de control interno. En este estudio, aspectos actitudinales propios de la etapa de vida en la que están estos estudiantes, como la búsqueda de la independencia y el desarrollo personal estarían acorde con lo planteado por Ajzen (1987). La intención emprendedora también está dada por la confianza que tienen los estudiantes en su capacidad de emprender nuevas acciones. La percepción de control de comportamiento ayuda a los estudiantes a determinar actividades y entornos, así como la persistencia, los patrones de pensamiento y las reacciones emocionales, cuando el individuo enfrenta obstáculos, siendo personas más proactivas y aumentando así 
la sensibilidad para la detección de nuevas oportunidades, lo cual es una precondición para emprender (Van Gelderen et al., 2008). En particular para este estudio, las actitudes personales hacia el emprendimiento tienen un efecto mayor sobre la intención emprendedora, que la percepción de control de comportamiento. En esta etapa de desarrollo de formación, la intención emprendedora estará determinada en mayor medida por la conveniencia de desarrollar o llevar a cabo una carrera empresarial que por la facilidad y/o dificultad que este accionar implique. De esta manera se valida el modelo de intención emprendedora basado en la TCP.

Esta investigación provee evidencia empírica que soporta la inclusión de la exposición al emprendimiento (Peterman y Kennedy, 2003). Se evidencia que someter frecuentemente a los estudiantes a actividades relacionadas con emprendimiento tiene un efecto positivo y directo sobre los determinantes de la intención emprendedora. Exponer a los estudiantes frecuentemente a este tipo de actividades podría impactar las actitudes personales hacia el emprendimiento.

El efecto de exposición impactaría las expectativas y creencias personales sobre los resultados derivados de la conducta, como la riqueza personal, el estrés, la autonomía y los beneficios para la comunidad (Shapero y Sokol, 1982). El efecto de exposición tambien impacta la percepción de las normas subjetivas del estudiante que tienen que ver con lo que piensa la gente importante en la vida del estudiante acerca de que este tenga una conducta emprendedora. La mera exposición a actividades relacionadas con el emprendimiento puede influenciar las percepciones del estudiante sobre las normas culturales/sociales cuando dicha exposición viene acompañada de una consecuencia adversa nula. Dicha exposición comunica una respuesta de que el entorno actual es seguro y por lo tanto hay un cambio en la percepción de la norma subjetiva. De igual manera, la percepción de control de comportamiento se ve influenciado por el efecto de exposición. Las actividades relacionadas con el emprendimiento plantean una mejora en la percepción de los estudiantes que les ayuda a determinar actividades y entornos, así como la persistencia, los patrones de pensamiento y las reacciones emocionales, cuando el individuo enfrente obstaculos.

Sería interesante que en próximos estudios se continue explorando la conexión entre la teoria del comportamiento planificado y la teoría de efectos de exposición, dado que proponen un camino para la formación en emprendimiento en estudiantes de colegio. El resultado de este estudio evidencia que más allá de proponer una cátedra específica en emprendimiento en los programas de los colegios de educacion básica y media, la 
formación hacia una cultura emprendedora puede darse en cualquier espacio académico y/o extracurricular, mediante el desarrollo de actividades relacionadas con el emprendimiento que de manera frecuente exponga a los estudiantes hacia las mismas. Este estudio contribuye a darle validez y solidez al modelo de intención basado en la teoría de comportamiento planificado teniendo en cuenta que existe poca evidencia empírica en estudiantes de colegio y en regiones con aspectos culturales propios latinoamericanos.

El presente estudio también tiene limitaciones: los datos obtenidos son transversales; un estudio longitudinal podría brindar mayor poder explicativo de validación a la investigación. Además los hallazgos se mantienen específicamente dentro de las características de la muestra, que corresponde a una región colombiana, con lo cual se debe ser cuidadoso en la extrapolación de los resultados a nivel nacional. Por otra parte, se propone que estudios futuros contemplen en la medición de la percepción de control de comportamiento los aspectos situacionales (externos) que complementan los personales (internos) como lo establece Azjen (1991). Finalmente, existen aún algunas limitantes frente al uso de las escalas de intención del comportamiento, como las diseñadas por Ajzen y Fishbein (1980), pues algunos autores señalan que estas miden las expectativas del comportamiento y no necesariamente la intención real (Davis y Warshaw, 1992).

\section{Referencias}

Ajzen, I., and Fishbein, M. (1980). Understanding attitudes and predicting social behaviour. Englewood Cliffs, NJ: Prentice-Hall.

Ajzen, I. (1987). Attitudes, trait and actions: Dispositional prediction of behavior in personality and social psychology. In L. Berkowitz (ed.). Advances in Experimental Social Psychology, 1-63.

Ajzen, I. (1991). The theory of planned behavior. Organizational behavior and human decision processes, 50, 179-211.

Armitage, C., and Conner, M. (2001). Efficacy of the theory of planned behaviour: A meta-analytic review. British Journal of Social Psychology, 40 (4), 471-499.

Baggozi, R., Baumgartner, H., and Yi, Y. (1989). An investigation into the role of intentions as mediators of the attitude-behavior relationship. Journal of Economic Psychology, 10 (1), 35-62.

Bandura, A. (1982). Self-efficacy mechanism in human agency. American Psychologist, 37 (2), 122-147.

Bandura, A. (1986). Social foundations of thought and action. Englewood Cliffs, NJ: Prentice-Hall. 
Bird, B. (1988). Implementing entrepreneurial ideas: the case for intention. Academy of Management Review, 13 (3), 442-453.

Bollen, K. A., and Davis, W. R. (2009). Causal indicator models: Identification, estimation, and testing. Structural Equation Modeling: A Multidisciplinary Journal, 16, 498-522.

Brannback, M., Carsrud, A., Elfvin, J., Kickul, J., and Krueger, N. (2007). Why replicate entrepreneurial intentionality studies? Prospects, perils and academic reality. available at: www.entrepreneurship.fiu.edu/downloads/publications/papers.

Cohen, J. (1988). Statistical power analysis for the behavioral sciences. Hillsdale, New Jersey: Lawrence Erlbaum Associates Inc.

Chin, W. (2010). How to write up and report PLS analyses. Handbook of Partial Least Squares (pp. 655-690). Berlin: Springer.

Chin, W. (1998). The partial least squares approach for structural equation modeling. Methodology for business and management. In G. Marcoulides. NJ: Lawrence Erlbaum Associates.

Davis, F. D., and Warshaw, P. R. (1992). What do intention scales measure? The Journal of General Psychology, 119, 391-407.

Diamantopoulos, A. (2001). Index construction with formative indicators: An alternative to scale development. Journal of Marketing Research, 38 (2), 269-277.

Diamantopoulos, A., Riefler, P., and Roth, K. P. (2008). Advancing formative measurement models. Journal of Business Research, 61, 1203-1218.

Donckels, R. (1991). Education and entrepreneuship experiences from secondary and university education in Belgium. Journal of Small Business and Entrepreneurship, 9 (1), 35-42.

Drennan, J., Kennedy, J., and Renfrow, P. (2005). Impact of childhood experiences on the development of entrepreneurial intentions. International Journal of Entrepreneurship \& Innovation, 6 (4), 231-238.

European Commission (2006). Entrepreneurship education in Europe: Fostering entrepreneurial mindsets through education and learning. In Final proceedings of the conference on entrepreneurship education, 0 slo.

Falk, R. (1992). A primer for soft modeling. University of Akron Press.

Fayolle, A., Gailly, B., and Lassas-Clerc, N. (2006). Assessing the impact of entrepreneurship education programmes: A new methodology. Journal of European Industrial Training, $30(9), 701-720$.

Filion, L. (1994). Ten steps to entrepreneurial teaching. Journal of small business and entrepreneurship, 11 (3), 68-78.

Fornell, C., and Bookstein, F. L. (1982). Two structural equation models: LISREL and PLS applied to consumer exit-voice theory. Journal of Marketing Research, 440.

Fornell, C. (1981). Structural equation models with unobservable variables and measurement error: Algebra and statistics. Journal of Marketing Research, 18 (3), 382-388. 
Gasse, Y. (1985). A strategy for the promotion and identification of potencial entrepreneurs at the secondary school level. Paper presented at the Frontiers of Entrepreneuship Research. Babson College: Wellesley.

Gefen, D., Straub, D. W., and Rigdon, E. E. (2011). An update and extension to SEM guidelines for admnistrative and social science research. Management Information Systems Quarterly, 35, iii-xiv.

Geisser, S. (1975). The predictive sample reuse method with applications. Journal of the American Statistical Association, 70 (350), 320-328.

Gist, M. (1987). Self-efficacy: Implications for organizational behavior and human resource management. Academy of Management Review, 12 (3), 472-485.

Godin, G., and Kok, G. (1996). The theory of planned behaviour: A review of its applications to health-related behaviors. American Journal of Health Promotion, 11 (2), 87-98.

Hagger, M. S., Chatzisarantis, N. L., and Biddle, S. J. (2002). A meta-analytic review of the theories of reasoned action and planned behavior in physical activity: Predictive validity and the contribution of additional variables. Journal of sport \& exercise psychology, 24 (1), 3-32.

Hair, J. R. (2011). PLS-SEM: Indeed a silver bullet. The Journal of Marketing Theory and Practice, $19(2), 139-152$.

Harris, M., and Gibson, S. (2008). Examining the entrepreneurial attitudes of US business students. Education + Training, 50 (7), 568-581.

Harrison, A. (1977). Mere exposure. In L. Berkowitz, Advances in Experimental Social Psychology. New York: Academic Press.

Herron, L., and Sapienza, H. (1992). The entrepreneur and the initiation of new venture launch activities. Entrepreneurship Theory and Practice, 17 (1), 49-55.

Hulland, J. (1999). Use of partial least squares (PLS) in strategic management research: A review of four recent studies. Strategic Management Journal, 20 (2), 195-204.

Jarvis, C. B., Mackenzie, S. B., and Podsakoff, P. M. (2003). A critical review of construct indicators and measurement model misspecification in marketing and consumer research. Journal of consumer research, 30, 199.

Julien, P. A. (2005). Emprendimiento regional y economía del conocimiento. Una metáfora de las novelas policiacas. Cali: Sello Editorial Javeriano.

Kolvereid, L. (1996). Prediction of employment status choice intention. Entrepreneurship Theory and Practice, 21 (1), 47-57.

Kolvereid, L., and Isaksen, E. (2006). New business start-up and subsequent entry into self-employment. Journal of Business Venturing, 21, 866-885.

Kourilsky, M. (1995). Entrepreneurship education: Opportunity in search of curriculum. Business Education Forum. October. 
Krueger, N. (1993). The impact of prior entrepreneurial exposure on perceptions of new venture feasibility and desirability. Entrepreneurship Theory and Practice, 18 (1), 5-21.

Krueger, N., Reilly, M., and Casrund, A. (2000). Competing models of entrepreneurial intentions. Journal of Business Venturing, 15 (5/6), 411-432.

Li, J., Wu, S., and Wu, L. (2008). The impact of higher education on entrepreneurial intentions of university students in China. Journal of Small Business and Enterprise Development, $15,752-774$.

Liñan, F. (2004). Intention based models of entrepreneurship education. Piccolla Impresa/Small Business, 3, 11-35.

Liñan, F., and Chen, Y. (2006). Testing the entrepreneurial intention model on a two-country sample. Working Paper 06/07, Department of Business Economics, Universitat Autonoma de Barcelona.

Mcclelland, D. C. (1973). Testing for competence rather than for intelligence. American psychologist, 28, 1.

Mcclelland, D. C., and Boyatzis, R. E. (1980). Opportunities for counselors from the competency assessment movement. The Personnel and Guidance Journal, 58, 368-372.

Mitchell, R., Busenitz, L., Bird, B., Gaglio, C., McMullen, J. et al. (2007). The central cuestion in entrepreneurial cognition research. Entrepreneurship Theory and Practice, 31 (1), 1-27.

Nunnally, J. (1978). Psychometric theory. New York: McGraw-Hill.

Peterman, N., and Kennedy, J. (2003). Enterprise education: Influencing students perceptions of entrepreneurship. Entrepreneurship Theory and Practice, 17, 129-144.

Reinartz, W., Haenlein, M., and Henseler, J. (2009). An empirical comparison of the efficacy of covariance-based and variance-based SEM. International Journal of Research in Marketing, 26, 332.

Rhodes, R. E., and Courneya, K. S. (2003). Investigating multiple components of attitude, subjective norm, and perceived control: An examination of the theory of planned behaviour in the exercise domain. British Journal of Social Psychology, 42, 129-146.

Ringle, C. W. (2005). SmartPLS 2.0 (M3) Beta. Hamburg: http://www.smartpls.de

Shapero, A., and Sokol, L. (1982). The social dimensions of entrepreneurship. In Encyclopedia of Entrepreneurship (pp. 72-90). Englewood Cliffs, NJ: Prentice-Hall.

Sheppard, B. H, Hartwick, J., and Warshaw, P. R. (1988). The theory of reasoned action: A meta-analysis of past research with recommendations for modifications and future research. Journal of consumer research, 15 (3), 325-343.

Souitaris, V., Zerbinati, S., and Al-Laham, A. (2007). Do entrepreneurship programmes raise entrepreneurial intention of science? The effect of learning, inspiration and resources. Journal of Business Venturing, 22 (4), 566-591. 
Sparks, P., Shepherd, R., Wieringa, N., and Zimmermans, N. (1995). Perceived behavioural control, unrealistic optimism and dietary change: An exploratory study. Appetite, 24 (3), 243-255.

Stone, M. (1974). Cross-validatory choice and assessment of statistical predictions. Journal of the Royal Statistical Society. Series B, 36 (2), 111-147.

Sutton, S. (1998). Predicting and explaining intentions and behavior: How well are we doing? Journal of applied social psychology, 28, 1317-1338.

Tkachev, A., and Kolvereid, L. (1999). Self-employment intentions among Russian students. Entrepreneurship and Regional Development, 11 (3), 269-280.

Van Den Putte, B. (1991). 20 years of the theory of reasoned action of Fishbein and Ajzen: A meta-analysis. Unpublished manuscript, University of Amsterdam.

Van Gelderen, M., Brand, M., Van Praag, M., Bodewes, W., Poutsma, E., and Van Gils, A. (2008). Explaining entrepreneurial intentions by means of the theory of planned behaviour. $\mathrm{Ca}$ reer Development International, 13 (6), 538-559.

Van Praag y Versloot. (2007). What is the value of entrepreneurship? A review of recent research. Small Business Economics, 4 (29), 351-382.

Veciana, J., Aponte, M., and Urbano, D. (2005). University student's attitudes towards entrepreneurship: a two countries comparison. International Entrepreneurship and Management Journal, 1, 165-182.

Wood, R., and Bandura, A. (1989). Social cognitive theory of organizational management. Academy of Management Review, 14 (3), 361-384.

$\mathrm{Wu}, \mathrm{S}$., and Wu, L. (2008). The impact of higher education on entrepreneurial intentions of university students in China. Journal of Small Business and Enterprise Development, 15 (4), 752-774.

Zajonc, R. (1968). The attitudinal effects of mere exposure. Journal of Personality and Social Psychology Monograph, 9 (2), 1-27.

Zajonc, R. (2001). Mere exposure: A gateway to the subliminal. Current Directions in Psychological Science, 10 (6), 224-228.

\section{Anexo 1. Preguntas utilizadas en la encuesta}

\section{Actitudes}

Estoy dispuesto a hacer lo necesario para ser emprendedor.

Si tuviese la oportunidad y los recursos, me gustaría crear una empresa.

Entre varias opciones, preferiría ser emprendedor.

Voy a hacer todo lo posible para iniciar y dirigir mi propia empresa. 


\section{Normas subjetivas}

\section{Creencia normativa}

Opinión con respecto a ser empresario de mis padres. Opinión con respecto a ser empresario de mis hermanos. Opinión con respecto a ser empresario de mis abuelos. Opinión con respecto a ser empresario de mis parientes. Opinión con respecto a ser empresario de mis amigos cercanos. Opinión con respecto a ser empresario de mis profesores. Opinión con respecto a ser empresario de mis conocidos. Opinión con respecto a ser empresario de otros.

\section{Motivación para cumplir}

Importancia de la opinión en tu decisión de ser empresario de mis padres. Importancia con respecto a ser empresario de mis hermanos. Importancia con respecto a ser empresario de mis abuelos. Importancia con respecto a ser empresario de mis parientes. Importancia con respecto a ser empresario de mis amigos cercanos. Importancia con respecto a ser empresario de mis profesores. Importancia con respecto a ser empresario de mis conocidos. Importancia con respecto a ser empresario de otros.

\section{PBC}

Estoy capacitado para proponer soluciones novedosas en situaciones reales e identifico alternativas que contribuyen a mi comunidad.

Soy capaz de motivar a las personas para realizar un proyecto, logrando la confianza del equipo de trabajo.

Estoy capacitado para tomar decisiones de manera rápida para enfrentar problemas y alcanzar las metas.

\section{Intención}

Tengo claro que voy a crear una empresa cuyos productos o servicios sean novedosos en el mercado. 
Tengo claro que voy a crear una empresa que utiliza procesos y equipos novedosos.

Tengo claros los pasos que debo dar para cumplir con mi objetivo de crear mi propia empresa.

\section{Exposición}

Frecuencia con la que empleo parte de mi tiempo aprendiendo sobre cómo crear mi propia empresa.

Frecuencia con la que investigo para reconocer oportunidades de crear empresa.

Frecuencia con la que resuelvo problemas que involucran cálculos financieros.

Frecuencia con la que trabajo en proyectos que involucran actividades empresariales. 
